\title{
Is Easy Access Related to Better Life? Walkability and Overlapping of Personal and Communal Identity as Predictors of Quality of Life
}

\author{
Michał Jaśkiewicz•Tomasz Besta
}

Received: 31 October 2012 / Accepted: 23 May 2013 / Published online: 13 June 2013

(C) The Author(s) 2013. This article is published with open access at Springerlink.com

\begin{abstract}
Two studies $(N=190$ and $N=447)$ were conducted to investigate the link between living in walkable neighborhoods and satisfaction with life in a city. Additionally, we explore possible mediators of this relationship. In both studies walkability was a significant predictor of perceived quality of life in a city, and overlap between personal and communal identity (in Study 1 and Study 2) and city identification (Study 2) were mediators of the walkability-quality of life relation. Implications for research on environmental qualities of neighborhoods and on selfconcept and communal identity are discussed.
\end{abstract}

Keywords Walkability. Quality of life - City identification · Identity fusion · Mediations

Quality of life and neighborhood satisfaction were found to be related to structural aspects of the environment (Amerigo and Aragones 1997; Galster 1987; Lipsetz 2001; Lévy-Leboyer, and Ratiu 1993). For example, Hur et al. (2010) found that the perception of a neighborhood as one with open space had the highest correlation with overall neighborhood satisfaction. There was evidence of a correlation between neighborhood characteristics (such as green space) and physical and mental health exist as well (De Vries et al. 2003; Maas et al. 2006). Humans also recognize regions as safe or not based on openness and enclosure of space (Appleton 1975; Gibson 1979).

Recent research on walkability highlighted the relationship between perception of how walking-friendly a given area is and the quality of life. The relationship was attributed to a number of factors, e.g., the benefits of physical exercise, impact on social capital, and accessibility of places that allow for relationship maintenance (Gill et al. 2010; Jeffres et al. 2009; Leyden 2003; Rogers et al. 2011). Because of the consequences

\footnotetext{
M. Jaśkiewicz $(\bowtie) \cdot$ T. Besta

Institute of Psychology, University of Gdansk, ul. Bażyńskiego 4, 80-952 Gdańsk, Poland

e-mail: psymj@ug.edu.pl

URL: http://www.ug.edu.pl/en
} 
of living in a walkable neighborhood to social relations and physical and mental health, it is not surprising that a number of studies examined the impact of walkability and accessibility on various urban services related to human life. For example, Leyden et al. (2011) found that cities with easy access to convenient public transportation and to cultural and leisure amenities promote happiness. An aesthetically pleasing streetscape for walkers contributes to lower rates of disorder and crime (Foster et al. 2011), whereas the fear of crime lessens the quality of life (Sacco 1993). Researchers also found associations between perceived physical environmental attributes (e.g., walkability perceptions) and mental well-being and neighborhood satisfaction (Guite et al. 2006; Leslie and Cerin 2008).

Research on the structural aspects of city architecture has indicated their impact on residents' functioning and satisfaction with life in a given city. However, social and psychological variables also need to be taken into account. These modify the perception of the place of living and often act as a buffer against environmental stressors. However, objective urban design qualities are different from social and psychological variables that may affect how individuals react to place. The notion that objective variables influence residential satisfaction and neighborhood attachment through indirect pathways that include personal characteristics is not controversial, and has been under investigation in several studies (e.g., chemical sensitivity, Nordin et al. 2003). For example, study results indicate that a sense of neighborliness in the immediate surroundings can compensate for mediocre living conditions (LévyLeboyer and Ratiu 1993) and that people-environment congruity is crucial for understanding people's perception of their neighborhoods (Moser 2009). The level of place attachment (operationalized as a bond that a person develops with a place) and social capital are also indicated as predictors of engagement in local communities and interest in the history of the city (Lewicka 2005, 2008). Although all of these studies are important for our understanding of the relationship between city environment and psychological variables, we will concentrate on a possible mediator of the relationship between structural aspects of environment and quality of life, namely the overlap of personal and communal identity.

\section{Current Studies: Why Overlapping of Identities Matters}

Our studies focus on two attributes of perceived quality of life. The first is walkability. We aimed to test whether walkability correlates with higher quality of life in a city, that is, whether previous findings on this relationship can be replicated in a different cultural context. At the same time, we focused on identifying potential psychological mediators of this relationship. We concentrated on psychological variables, not on social and demographic ones, as there are numerous studies analyzing the role of demographic factors in a sense of quality of life. However, the aim of our study was to account for the psychological variables that may potentially mediate the influence of environmental factors such as walkability on perceived quality of life. We hoped that this approach would contribute to a fuller and more comprehensive understanding of the problem of subjectively perceived quality of life in the city. 
Research from cross-cultural and organizational psychology highlights the fit between a person and the environment as a factor that can influence people's quality of life. The basis for person-culture fit can be assessed in terms of the similarity between the person and the group in dimensions such as values, goals, and personality traits (Elfenbein and O'Reilly 2007). For example, Ratzlaff et al. (2000) proposed that the extent to which there was a discrepancy between one's personal values and one's perceived societal values will affect an individual's health and wellbeing. This hypothesis was partially confirmed by Lu (2006). In that study, people who were consistent with their societal culture declared higher subjective well-being than those who were not. Based on different theoretical approaches, research on person-culture match show that, when a person's personality matches the dominant personalities of other people in a culture, culture functions as an important amplifier of the positive effect of personality on self-esteem and subjective well-being at the individual level (Fulmer et al. 2010).

Moreover, recently yet another theoretical approach has concentrated on the overlap between individual and group characteristics. As a number of studies on the perceived consistency between personal and social identity indicate, individuals with personal and group identity fusion are more inclined to sacrifice for other group members and to display more pro-social behaviors (Swann et al. 2010). It seems that the identity fusion overlap framework can be applied in the analysis of relationships with the city and local community and can expand the knowledge about psychological mechanisms that mediate the relationship between structural aspects of the city and residents' feelings. In order to shift the analysis from "culture" to "city," we decided not to refer to the similarity of participants' goals and values to the goals and values attributed to the "average citizen" or to the goals and values perceived as dominant in a given city or community. In order to capture the aspect of citizens' identity, we decided to implement the measure of perceived overlap between personal identity - which includes a personal sense of the uniqueness of the self-and communal identity, which refers to the category of viewing "self as an inhabitant of this city." The greater overlap of the identities indicates a larger role of communal identity in guiding a person's attitudes and behaviors, and suggests that personal and communal identities can synergistically reinforce one another.

In order to introduce possible mediators of the relationship between walkability and quality of life, we employed two constructs that are currently applied and developed in the psychology of intergroup relations, namely group identification and identity fusion. In short, group identification is known to be a significant predictor of pro-group attitudes and in-group favoritism. Social identity theory (Tajfel \& Turner 1979) proposes that striving to develop a positive identity through highlighting the superiority of one's group is a universal human motivation. Studies conducted in the field of environmental psychology concluded that, in terms of social identity theory (Tajfel \& Turner 1979), place identifications are an addition to the existing range of identifications (Rollero and De Piccoli 2010). In the realm of environmental research, it was proved that individuals with low place identity were more annoyed by traffic noise (Jonah et al. 1981), and strong topological identity was linked to underestimation of the frequency of incivilities (Felonneau 2004). Social aspects of neighborhood and being the member of a community are also outlined as the important factors in one's residential environment. Moser et al. (2003) suggest 
that social ties that contribute to well-being and quality of life are also developed from local contacts. Place identification also leads to more positive perceptions of the place and its inhabitants (Rollero and De Piccoli 2010). These findings seem to be clear evidence that city identification should be considered as a mediator between objective physical variables and perceived quality of life in a city.

The second proposed psychological mediator is identity fusion, that is, a state in which personal and social identities overlap, and personal and group schema merge. For fused individuals, the self-other barrier is blurred and the group is regarded as equivalent to the personal self (Gómez et al. 2011; Swann et al. 2009). Research on identity fusion is complementary to the classical social identity theory and helps to explain inconsistencies in the results of the studies that were conducted in the social identity paradigm (Swann et al. 2009). Applying an identity fusion scale in the context of identification with the city allows us to capture the way in which perceived similarity between personal and communal identities will impact a sense of contentment, and whether it will mediate the relationship between walkability and perceived quality of life. Both group identification scale and identity fusion scale are related to strong group adherence, and whether identity fusion should be perceived as an independent psychological construct or as one of the measures of strong group identification is still under dispute. However, previous studies have confirmed that an identity fusion scale is more strongly related to the feeling of group agency and is a better predictor of pro-group attitudes and behaviors than some of the group identification measures (for review, see Swann et al. 2012). As it was not the purpose of the current studies to resolve this debate, we used both measures in order to explore their relationships with life satisfaction when referring to the context of the city.

Based on the above-mentioned studies, we formulated the following research goals: 1) to test whether walkability is a significant predictor of residents' overall quality of life in the city, and 2) to test whether the overlap between personal and communal identity and a person's identification with the city mediates the relationship between the structural aspects of neighborhood and quality of life in the city.

\section{Study 1}

Participants and Method

Residents of Tri-city in Poland (an agglomeration of Gdansk, Gdynia, Sopot, and the surrounding areas) participated in the study. The study was carried out via the Internet. An invitation to participate was posted on social media websites and Internet forums dedicated to news about local communities and housing estates. We received 190 questionnaires (109 from women, 81 from men). Mean age was $32.8(\mathrm{SD}=8.66)$. Due to missing data, the size of the samples in the analyses may vary. All measures were based on participants' subjective opinions. The set of questionnaires contained:

a) Walkability Scale-12 items (Cronbach $\alpha=0.89$ ). Walkability was a measure of how easy it is to get to places such as corner shops, the cinema, church, and the local pharmacy without a car. Participants responded to a five-point Likert scale anchored from very difficult (coded as 1) to very easy (coded as 5). 
b) Identity Fusion Scale - seven items. This was based on a scale by Gómez et al. (2011) that measures the overlap between personal and group identity in the context of place of living $(\alpha=0.93)$. The scale contains the following items: "My city is me"; "I am one with my city"; "I feel immersed in my city"; "I have a deep emotional bond with my city"; "I am strong because of my city"; "I will do more for my city than any other group members would do"; and "I make my city strong." A seven-point Likert scale was used to allow the individual to express how much they agree or disagree with a particular item.

c) Group Identification Scale. We applied a scale of six items that measures group identification $(\alpha=0.91)$ to items adapted to the context of the city (Mael and Ashforth 1992). This allowed us to test whether identity fusion is indeed strongly related to identification with the city and whether both constructs are significant mediators of the relationship between walkability and quality of life. Items of identification with the city scale included: "When someone criticizes my city, it feels like a personal insult"; "I am very interested in what others think about my city"; "When I talk about my city I usually say "we' rather than 'they",; "City successes are my successes"; "When someone praises my city, it feels like a personal compliment"; and "If a story in the media criticized my city I would feel embarrassed." Participants responded using a seven-point Likert scale.

d) Quality of Life in a City Scale - three items $(\alpha=0.92)$ : These included "I feel happy in my city"; "I feel fulfilled in my city"; and "I am glad to be living in my city." Participants used a seven-point Likert scale response format.

\section{Results}

Means, standard deviations, and correlational matrix of variables included in the model are displayed in Tables 1 and 2 .

In order to test whether identity variables and those related to the city are significant predictors of a sense of satisfaction, we applied a stepwise regression analysis. In the first step we entered the results of the walkability scale, in the second step, a fusion of personal and group identity was entered as well as identification with the city. Results are displayed in Table 3. In the first step, higher level of walkability

Table 1 Means (M) and standard deviations (SD) of variables included in the model

\begin{tabular}{|c|c|c|c|c|c|c|}
\hline & \multicolumn{3}{|c|}{ Study I } & \multicolumn{3}{|c|}{ Study II } \\
\hline & $M$ & $S D$ & $N$ & $M$ & $S D$ & $N$ \\
\hline Walkability (scale $1-5$ in study $1 ; 1-4$ in study 2 )* & 3.57 & 0.896 & 190 & 2.86 & 0.400 & 413 \\
\hline City Identification (scale 1-7) & 4.23 & 1.42 & 185 & 4.22 & 1.34 & 435 \\
\hline Identity Fusion (scale 1-7) & 3.77 & 1.44 & 180 & 3.77 & 1.33 & 430 \\
\hline Quality of Life in a City (scale $1-7)^{* *}$ & 4.81 & 1.42 & 190 & 4.76 & 1.06 & 447 \\
\hline
\end{tabular}

*Walkability scores was obtained by the different measures in each study. **Quality of life in a city was measured by 3 -items scale in a study I and by 5 -items scale in a study II 
Table 2 Correlation matrix

\begin{tabular}{|c|c|c|c|c|c|c|}
\hline & \multicolumn{2}{|c|}{ Walkability } & \multicolumn{2}{|c|}{ City Identification } & \multicolumn{2}{|c|}{ Identity Fusion } \\
\hline & Study I & Study II & Study I & Study II & Study I & Study II \\
\hline City Identification & 0.00 & 0.13 & & & & \\
\hline Identity Fusion & $0.18^{*}$ & $0.22 * * *$ & $0.76 * *+*$ & $0.71 * * *$ & & \\
\hline Quality of Life in a City & $0.16^{*}$ & $0.19 * * *$ & $0.46 * * *$ & $0.44 * * *$ & $0.53 * * *$ & $0.49 * * *$ \\
\hline
\end{tabular}

${ }^{*} p<0.05 ; * * * p<0.001$

turned out to be a significant predictor of quality of life in the city. When identity variables were entered in the second step, identity fusion became a significant predictor, while walkability ceased to be.

The results of the regression analysis indicate that it is in fact identity fusion that can be the mediator of the relationship between walkability and neighborhood satisfaction. In order to verify whether the results of the regression analysis would be confirmed by a mediation analysis, we conducted one with the use of a bootstrapping macro by Preacher and Hayes (2008). In this analysis, walkability was entered as the predictor, identity fusion and city identification were entered as mediators (we included an identification scale, although it was not a significant predictor in regression analyses, as the correlation between fusion and identification was significant and relatively high), and neighborhood satisfaction as the depended variable. Using the SPSS macro provided by Preacher and Hayes, we conducted a bootstrapping test for the model ( $\mathrm{n}$ boots $=5,000$ ). As can be seen in Fig. 1, mediation analyses indicated that identity fusion is a significant mediator of the proposed relationship (confidence intervals for identity fusion are above zero).

\section{Discussion}

In the first study, we were able to show that walkability is related to perceived quality of life and that this relationship is mediated by identity fusion. However, an important argument for replicating the study was that, to measure walkability, we used the authors' scale that asked about the ease of reaching services and culture sites. As

Table 3 Summary of hierarchical regression analysis for the prediction: Quality of life in the city in Study 1

\begin{tabular}{|c|c|c|c|c|}
\hline & & $R^{2}$ adj. & $B$ & $t$ \\
\hline \multirow[t]{2}{*}{ Step 1} & & 0.021 & & \\
\hline & Walkability & & 0.164 & $2.204 *$ \\
\hline \multirow[t]{4}{*}{ Step 2} & & 0.282 & & \\
\hline & Walkability & & 0.100 & 1.513 \\
\hline & Identity fusion & & 0.376 & $3.665^{* * *}$ \\
\hline & City identification & & 0.173 & $1.709^{\wedge}$ \\
\hline
\end{tabular}

${ }^{\wedge} p=0.089 * p<0.05 * * * p<0.001 ; \Delta R^{2}=0.267 ; p<0.001$ 
A)

Model: $95 \%$ confidence intervals; boots $=5000 ; \mathrm{N}=178$;

Bias corrected confidence intervals: FUSION $=.0042$ to .0697 ; IDENTIFICATION $=-.0102$ to .

0168;

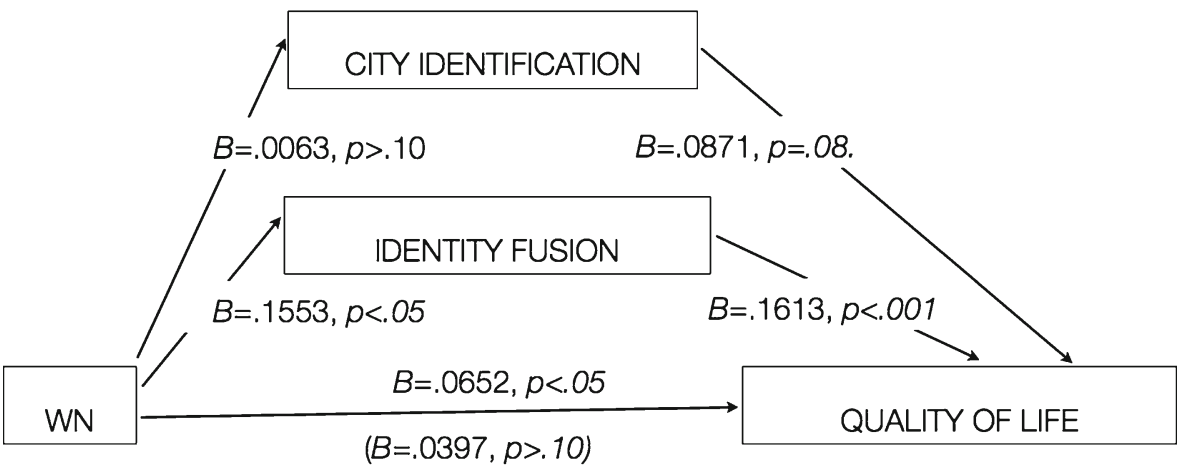

Fig. 1 Summary of mediation analyses on quality of life in Study 1

such, we have decided to increase the size of the research sample in the second study and use the Polish adaptation of the validated in many cultures' NEWS scale.

\section{Study 2}

Participants and Method

In order to obtain a diversified sample, we asked students to send a link to the study to their friends and adult members of their families. As a result, 447 people participated in the study (303 women, 144 men). Mean age was $23.7(S D=6.70)$. Due to missing data, the number of participants in the conducted analysis may vary. Similarly to Study 1, all measures were based on the participants' subjective attitudes and responses to the questions raised.

The set of questionnaires contained:

a) A Polish version of the NEWS (Neighborhood Environment Walkability Survey) scale (Cerin et al. 2006) -51 items (reliability $\alpha=0.88$ ): The scale consists of 12 factors: residential density, land-use mix-diversity, land-use mix-access, street connectivity, infrastructure and safety for walking, aesthetics, traffic hazards, crime, lack of parking, lack of cul-de-sacs, hilliness, and physical barriers. For example, in a residential density subscale, participants estimated how common apartments or condos are in their immediate neighborhood (e.g., apartments of 1-3 stories, apartments of 4-6 stories, etc.). Responses may vary from none (coded as 1) to all (coded as 5). On a land-use mix-diversity subscale, participants estimated walking distance to facilities (e.g., fruit and vegetable market, park) in minutes. The response format was as follows: (1) $31 \mathrm{~min}+$, (2) 21-30 min, (3) 11-20 min, (4) 6-11 min, and (5) 1- 
$5 \mathrm{~min}$. In the remaining subscales, participants were asked to respond to statements such as: "There are many attractive natural sights in my neighborhood" (aesthetic factor) and "There are many alternative routes for getting from place to place in my neighborhood" (street connectivity factor). Participants responded on a four-point Likert scale anchored from "strongly disagree" (1) to "strongly agree" (4).

b) Identity Fusion Scale. This consists of seven items based on a scale (Gómez et al. 2011) that measures the overlap between personal identity and group identity in the context of place of residence (reliability $\alpha=0.94$ ).

c) Identification with the City Scale. This is based on Mael and Ashforth's (1992) group identity scale $(\alpha=0.91)$.

d) Quality of Life in a City scale - five items $(\alpha=0.83)$. Compared to Study 1 , two items were added: "I would not move to another city" and "In my city good things tend to happen more often than bad ones." Similar to Study 1, participants used seven-point Likert scale response format.

\section{Results}

Again, we started by verifying whether the variables related to the city and the variables related to identity are significant predictors of quality of life in a city. We conducted a stepwise regression analysis, with walkability entered in Step 1 and communal and personal identity fusion, as well as city identification added in Step 2. The results are displayed in Table 4. Similarly to the previous study, in Step 1, walkability turned out to be a significant predictor of neighborhood satisfaction. In Step 2, after we had entered the psychological variables, the predictive power of walkability dropped, and both identity variables (fusion and identification) turned out to be significantly related to the sense of quality of life in a city.

In order to verify whether regression analysis results would once again be confirmed by mediation analysis, we employed the bootstrapping macro by Preacher and Hayes (2008). Walkability was entered as the predictor, identity fusion and city identification as mediators, and neighborhood satisfaction as the dependent variable. Using the SPSS macro, we conducted a bootstrapping test for the model ( $\mathrm{n}$ boots $=5,000)$. As can be seen in Fig. 2, both psychological variables were significant mediators of the relationship between a walking-friendly neighborhood and quality of life in a city.

Table 4 Summary of hierarchical regression analysis for the prediction: Quality of life in the city in Study 2

\begin{tabular}{lllll}
\hline & & $R^{2}$ adj. & $B$ & $t$ \\
\hline Step 1 & & 0.041 & & \\
& Walkability & & 0.207 & $4.439^{* * *}$ \\
Step 2 & Walkability & 0.260 & & $2.278^{*}$ \\
& Identity fusion & & 0.096 & $5.327^{* * *}$ \\
& City identification & & 0.313 & $3.658^{* * *}$ \\
\hline
\end{tabular}

$* p<0.05 * * * p<0.001 ; \Delta R^{2}=0.223 ; p<0.001$ 
We also tested whether it is the walkability that mediates the relationship between fusion and quality of life in a city. We found that controlling for the NEWS scores does not change the strength of the relationship between fusion and satisfaction $(B=2.848, p$ $<0.001$ before entering walkability and $B=2.748, p<0.001$ after). We obtained similar results in the first study (before entering walkability, $B=2.264$ and $B=2.203$ after; in both cases $p<0.001$ ). As such, it is the identity fusion that is the significant mediator of the relationship between walkability and quality of life in a city (while walkability is not the mediator of the relationship between fusion and satisfaction).

\section{Discussion}

In both studies, walkability turned out to be a significant predictor of perceived quality of life in a city. As such, previous studies concerning a relationship between pedestrian-friendly spaces and well-being related variables were confirmed (Leyden 2003; Leyden et al. 2011; Rogers et al. 2011). At the same time, our studies show that the extent to which the city is central to self-concept can be the mediating variable.

Identity fusion mediates the relationship between walkability and quality of life in a city, which suggests that more pedestrian-friendly neighborhoods foster greater integration between self-categorization in both private and personal dimensions, as well as in dimensions connecting the self with the city and the neighborhood in which a person lives. Additionally, scores on the identification with the city scale were a significant mediator of the relationship between walkability and quality of life (in Study 2), which shows that those two psychological constructs (fusion and identification) yield similar predictions, at least in the context of a city as used in this study.

As to mean scores on measures of city's adherence, it should be noted that they fall below the scales arithmetic means. It is not usual result, as place attachment scores

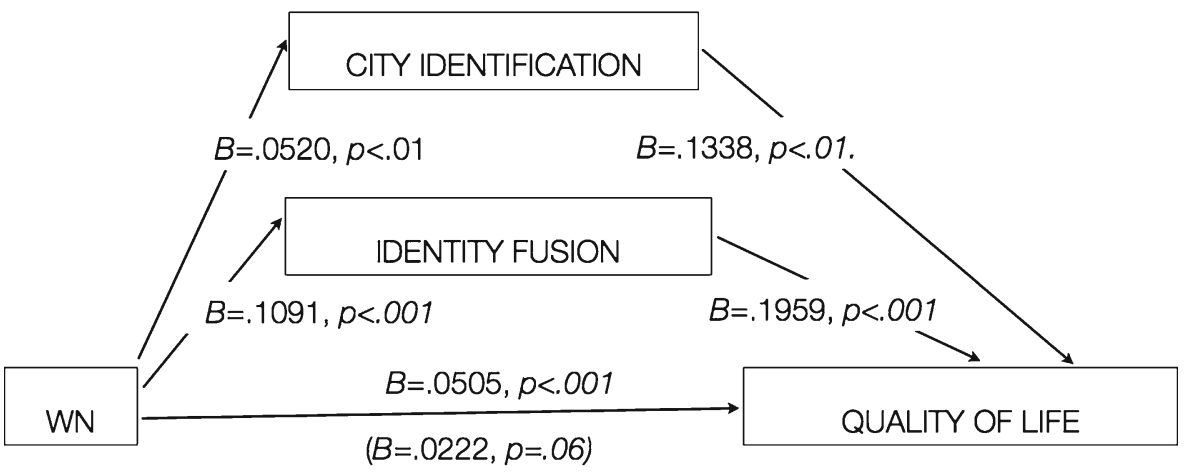

Fig. 2 Summary of mediation analyses on quality of life in Study 2 
assessed by city-specific measures tend to fall above it. The reason for this could be the fact, that both scales we used (city identification and identity fusion) were direct transference of the measures of relationship to the group. On the negative side of our scales' selection should be highlighted that those scales are not constructed to tap specific aspects of the city/place attachment. On the bright side however, our choice of assessment tools allow for comparison of the strength of ties to different social groups and entities. In this view, for example, we can emphasized that mean scores of personal and communal identity fusion obtained in our research (that is $M=3.77$ ) are higher then mean scores of identity fusion in the context of country (e.g. $M=$ 3.08) and religion (e.g. $M=2.71$ ) but lower than fusion of personal and family identity (e.g. $M=4.84$ ) (all mean scores for identity fusion in different contexts are from Besta 2013).

Evidently, the presented studies are correlational and do not show the impact of the place of living on city identification and on the positive evaluation of living in a particular area. However, we can still consider the potential mechanisms that might explain the obtained results of the mediation analyses. Why would walkability promote a better city-person fit and an individual identity fusion with perception of oneself as a city inhabitant?

Previous research found that people living in more walkable neighborhoods are more eager to volunteer, work on community projects, and meet with friends (Rogers et al. 2011). People who live in walkable neighborhoods are more likely to know their neighbors, participate politically, trust each other, and be socially engaged (Leyden 2003). As Leyden (Ibid.) argued, a pedestrianoriented neighborhood enables residents to interact. These spontaneous interactions can help to strengthen a sense of connection between people and the place where they live. For most residents, such contacts provide a comfortable feeling associated with satisfying the need for predictability and familiarity. We think that these important suggestions related to walkable neighborhoods are partially confirmed in our studies: pedestrian-oriented neighborhoods may indirectly influence quality of life through a stronger inclusion of communal identity in the self-concept. Consequently, the positive social outcomes of living in more walkable areas observed in a some studies may be attributed to a perception of "oneself as a community member" and to inclusion of this social role in one's self-definition, which could lead to more fused identities. As identity fusion is related, for example, to more donations to charity in order to help one's social group, it indicates that fusion is associated with overt and positive behavior toward in-group members (in our case: people living in the same city) (Swann et al. 2010). Obviously more studies are needed on the mediation role of psychological variables in the relationship between structural aspects of environment and perceived quality of life in a city, but mutual reciprocity and positive interactions with fellow citizens could result in higher overall neighborhood satisfaction.

To sum up, our research replicated and extended previous findings on the association between walkability and various well-being measures. We introduce a potential mediator of this relationship, that is, strong communal identity measured by identity fusion scale or by identification with a city scale. The present findings imply that not only is more research on walkability and well-being needed, but also that studies 
concerning the influence of living in a walkable neighborhood on one's self-concept and identity are worth pursuing.

Open Access This article is distributed under the terms of the Creative Commons Attribution License which permits any use, distribution, and reproduction in any medium, provided the original author(s) and the source are credited.

\section{References}

Amerigo, M., \& Aragones, J. I. (1997). A theoretical and methodological approach to the study of residential satisfaction. Journal of Environmental Psychology, 17(1), 47-57.

Appleton, J. (1975). The experience of landscape. London: William Clowes.

Besta, T. (2013). Identity fusion in various group contexts. Gdansk: Unpublished manuscript. Institute of Psychology, University of Gdansk.

Cerin, E., Saelens, B. E., Sallis, J. F., \& Frank, L. D. (2006). Neighborhood environment walkability scale: Validity and development of a short form. Medicine \& Science in Sports \& Exercise, 38(9), 1682-1691.

Elfenbein, H. A., \& O'Reilly, C. A. (2007). Fitting in: The effects of relational demography and personculture fit on group process and performance. Group \& Organization Management, 32(1), 109-142.

Felonneau, M.-L. (2004). Love and loathing of the city: Urbanophilia and urbanophobia, topological identity and perceived incivilities. Journal of Environmental Psychology, 24, 43-52. doi:10.1016/ S0272-4944(03)00049-5.

Foster, S. A., Giles-Corti, B., \& Knuiman, M. W. (2011). Creating safe walkable streetscapes: Does house design and upkeep discourage incivilities in suburban neighbourhoods? Journal of Environmental Psychology, 31(1), 79-88.

Fulmer, C. A., Gelfand, M. J., Kruglanski, A. W., Kim-Prieto, C., Diener, E., Pierro, A., \& Higgins, E. T. (2010). On "feeling right" in cultural contexts: How person-culture match affects self-esteem and subjective well-being. Psychological Science, 21(11), 1563-1569. doi:10.1177/0956797610384742.

Galster, G. (1987). Identifying the correlates of dwelling satisfaction. Environment and Behavior, 19(5), $539-568$.

Gómez, A., Brooks, M. L., Buhrmester, M. D., Vázquez, A., Jetten, J., \& Swann, W. B., Jr. (2011). On the nature of identity fusion: Insights into the construct and a new measure. Journal of Personality and Social Psychology, 100(5), 918-933.

Gibson, J. (1979). The ecological approach to visual perception. Hillsdale: Lawrence Erlbaum.

Gill, D. L., Chang, Y., Murphy, K. M., Speed, K. M., Hammond, C. C., Rodriguez, E. A., Lyu, M., \& Shang, Y. (2010). Quality of life assessment for physical activity and health promotion. Applied Research in Quality of Life, 6(2), 181-200.

Hur, M., Nasar, J. L., \& Chun, B. (2010). Neighborhood satisfaction, physical and perceived naturalness and openness. Journal of Environmental Psychology, 30(1), 52-59.

Guite, H. F., Clark, C., \& Ackrill, G. (2006). The impact of the physical and urban environment on mental well-being. Public Health, 120(12), 1117-1126.

Jonah, B. A., Bradley, J. S., \& Dawson, N. E. (1981). Predicting individual subjective responses to traffic noise. Journal of Applied Psychology, 66(4), 409-501.

Jeffres, L. W., Bracken, C. C., Jian, G., \& Casey, M. F. (2009). The impact of third places on community quality of life. Applied Research in Quality of Life, 4(4), 333-345.

Leslie, E., \& Cerin, E. (2008). Are perceptions of the local environment related to neighbourhood satisfaction and mental health in adults? Preventive Medicine, 47(3), 273-278.

Lévy-Leboyer, C., \& Ratiu, E. (1993). The need for space and residential satisfaction. Architecture \& Comportement/Architecture \& Behaviour, 9, 475-490.

Lewicka, M. (2005). Ways to make people active: The role of place attachment, cultural capital, and neighborhood ties. Journal of Environmental Psychology, 25(4), 381-395.

Lewicka, M. (2008). Place attachment, place identity, and place memory: Restoring the forgotten city past. Journal of Environmental Psychology, 28(3), 209-231.

Leyden, K. M. (2003). Social capital and the built environment: The importance of walkable neighborhoods. American Journal of Public Health, 93(9), 1546-1551.

Leyden, K. M., Goldberg, A., \& Michelbach, P. (2011). Understanding the pursuit of happiness in ten major cities. Urban Affairs Review, 47(6), 861-888. 
Lipsetz, D. A. (2001). Residential satisfaction: Identifying the differences between suburbanites and urbanites. Ohio State University.

Lu, L. (2006). "Cultural fit": Individual and societal discrepancies in values, beliefs, and subjective wellbeing. Journal of Social Psychology, 146(2), 203-221.

Mael, F., \& Ashforth, B. E. (1992). Alumni and their alma mater: A partial test of the reformulated model of organizational identification. Journal of Organizational Behavior, 13(2), 103-123.

Maas, J., Verheij, R. A., Groenewegen, P. P., de Vries, S., \& Spreeuwenberg, P. (2006). Green space, urbanity, and health: How strong is the relation? Journal of Epidemiology and Community Health, 60, $587-592$.

Moser, G. (2009). Quality of life and sustainability: Toward person-environment congruity. Journal of Environmental Psychology, 29(3), 351-357.

Moser, G., Legendre, A., \& Ratiu, E. (2003). Citydwellers' relationship networks: Patterns of adjustment to urban constraints. In R. Garcia Mira, J. M. Sabucedo Cameselle, \& J. Romay Martinez (Eds.), Culture, environmental action and sustainability (pp. 161-170). Ashland: Hogrefe \& Huber Publishers.

Nordin, S., Millqvist, E., Löwhagen, O., \& Bende, M. (2003). The chemical sensitivity scale: Psychometric properties and comparison with the noise sensitivity scale. Journal of Environmental Psychology, 23, 359-367.

Preacher, K. J., \& Hayes, A. F. (2008). Asymptotic and resampling strategies for assessing and comparing indirect effects in multiple mediator models. Behavior Research Methods, 40(3), 879-891.

Ratzlaff, C., Matsumoto, D. K. N., Raroque, J., \& Ray, R. (2000). Individual psychological culture and subjective well-being. In E. Diener \& E. M. Sul (Eds.), Culture and subjective well-being (pp. 37-60). Cambridge: MIT Press.

Rogers, S. H., Halstead, J. M., Gardner, K. H., \& Carlson, C. H. (2011). Examining walkability and social capital as indicators of quality of life at the municipal and neighborhood scales. Applied Research in Quality of Life, 6(2), 201-213.

Rollero, C., \& De Piccoli, N. (2010). Place attachment, identification and environmental perception: An empirical study. Journal of Environmental Psychology, 30(2), 198-205.

Sacco, V. F. (1993). Social support and the fear of crime. Canadian Journal of Criminology, 35, 187-196.

Swann, W. B., Jr., Gómez, A., Huici, C., Morales, J. F., \& Hixon, J. G. (2010). Identity fusion and selfsacrifice: Arousal as a catalyst of pro-group fighting, dying, and helping behavior. Journal of Personality and Social Psychology, 99(5), 824-841.

Swann, W. B., Jr., Gómez, A., Seyle, D. C., Morales, J. F., \& Huici, C. (2009). Identity fusion: The interplay of personal and social identities in extreme group behavior. Journal of Personality and Social Psychology, 96(5), 995-1011.

Swann, W. B., Jr., Jetten, J., Gómez, Á., Whitehouse, H., \& Bastian, B. (2012). When group membership gets personal: A theory of identity fusion. Psychological Review, 119(3), 441-456. doi:10.1037/a0028589.

Tajfel, H., \& Turner, J. C. (1979). An intergrative theory of intergroup conflict. In W. G. Austin \& S. Worchel (Eds.), The Social Psychology of Intergroup Relations (pp. 33-47). Monterey, CA: Brooks/ Cole.

de Vries, S., Verheij, R. A., Groenewegen, P. P., \& Spreeuwenberg, P. (2003). Natural environments - healthy environments? An exploratory analysis of the relationship between green space and health. Environment and Planning A, 35, 1717-1731. 